13. W. M. Rohsenow and H. CHOI, Heat, Mass and Momentum Transfer, p. 717. Prentice Hall, Englewood Cliffs, (1963).

14. P. C. LU, A theoretical investigation of combined free and forced convection heat generating laminar flow inside vertical pipes with prescribed wall temperatures, M.S. Thesis, Kansas State College, Manhattan, Kansas (1959).

15. N. W. McLachlan, Bessel Functions for Engineers. Oxford University Press, England (1934).

Int. I. Heat Mass Transfer. Vol. 14, pp. 495-498. Pergamon Press 1971. Printed in Great Britain

\title{
HEAT TRANSFER IN THE LAMINAR CREEPING FLOW BETWEEN PARALLEL CIRCULAR DISKS WITH ECCENTRIC INLET
}

\author{
S. C. TANG \\ Ford Motor Company, Dearborn, Michigan 48121, U.S.A. \\ J. R. CAIRNS and T. Y. NA \\ University of Michigan—Dearborn Campus, Dearborn, Michigan 48128, U.S.A.
}

(Received 8 April 1970 and in revised form 1 July 1970)

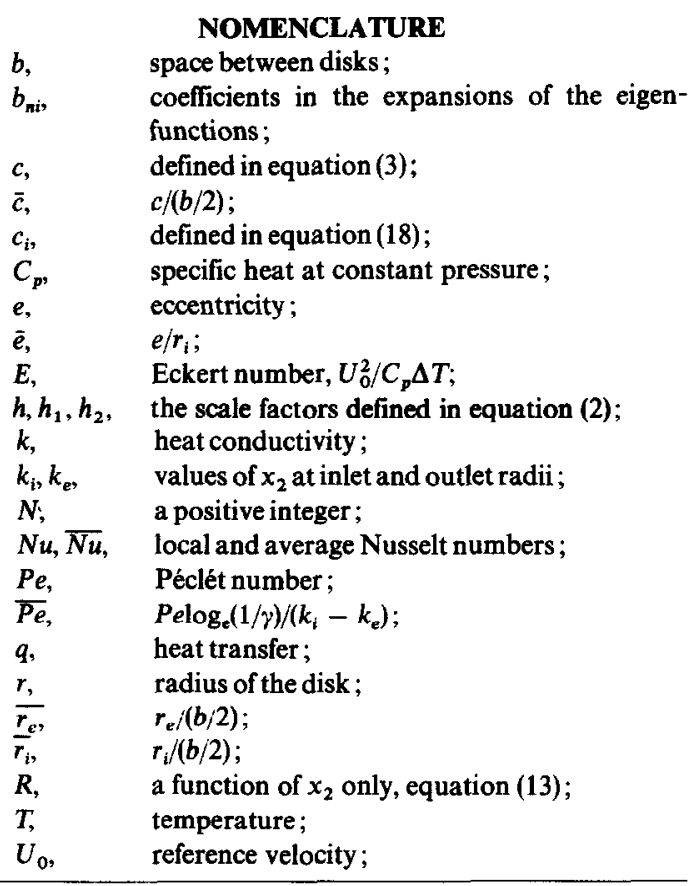

1. Senior Research Engineer, Scientific Research Staff, the Ford Motor Company, Dearborn, Michigan, 48121.

2. Professor and Chairman, Division of Engineering, the University of Michigan-Dearborn Campus, Dearborn, 48128.

3. Professor of Mechanical Engineering, the University of Michigan-Dearborn Campus, Dearborn, Michigan, 48128 .
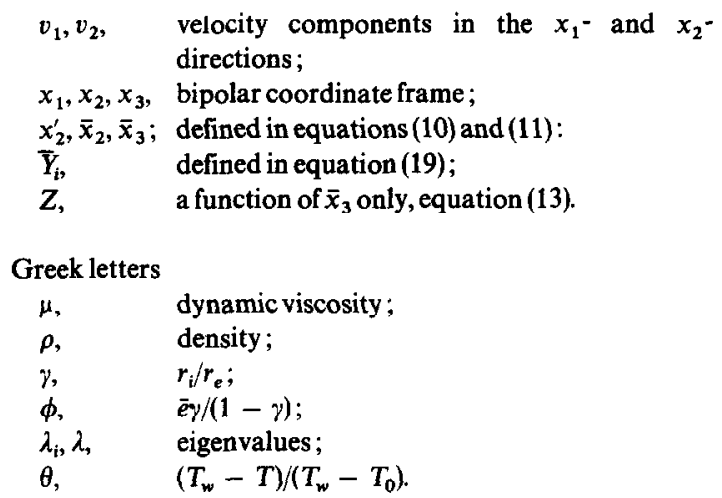

Subscripts $i, e$ and $w$ refer to conditions at inlet, exit and wall respectively.

\section{ANALYSIS}

THE ENRGY equation for the laminar, creeping flow of incompressible fluids between parallel circular disks with eccentric inlet (see Fig. 1) can be written, in bipolar coordinates, as

$$
\rho C_{p}\left(\frac{v_{1}}{h_{1}} \frac{\partial T}{\partial x_{1}}+\frac{v_{2}}{h_{2}} \frac{\partial T}{\partial x_{2}}\right)=k \frac{\partial^{2} T}{\partial x_{3}^{2}}
$$

subject to the boundary conditions:

$$
\begin{gathered}
T\left(x_{1}, x_{2}, b / 2\right)=T\left(x_{1}, x_{2},-b / 2\right)=T_{w} \\
T\left(x_{1}, k_{i}, x_{3}\right)=T_{0} .
\end{gathered}
$$

The viscous dissipation terms are neglected, which is justified for small Eckert numbers, i.e. $E\left(=\mathrm{U}_{m}^{2} / C_{p} \Delta T\right) \ll 1$. 

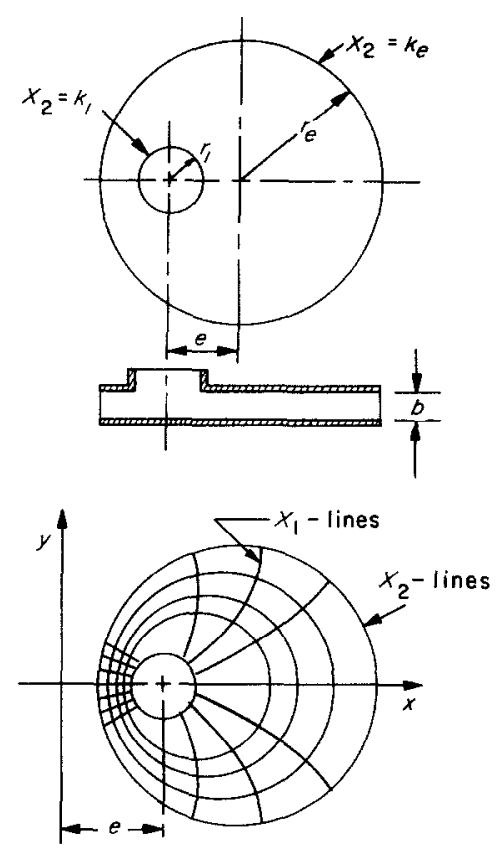

Fig. 1. Bipolar coordinates and the disks.

The conduction in $x_{1}$ - and $x_{2}$-directions are neglected in comparison with conduction in the $x_{3}$-direction. Also, the entrance effect can be neglected due to the extremely small clearance between the disks in such problems. The scale factors, $h_{1}$ and $h_{2}$, of the bipolar coordinates are defined by:

$$
h_{1}=h_{2}=h=\frac{C}{\cosh x_{2}-\cos x_{1}} .
$$

The coordinate plane, $x_{3}=0$, is parallel to and midway between the disk surfaces. The constant $C$ is related to the inner and outer radii, $r_{i}$ and $r_{e}$, and the eccentricity $e$, through the relation:

$$
C=r_{\imath} \sinh k_{\imath}=r_{e} \sinh k_{e}
$$

where

$$
\begin{aligned}
& k_{i}=\cosh ^{-1} \frac{1 \gamma\left(1+\phi^{2}\right)+\left(1-\phi^{2}\right)}{2 \phi} \\
& k_{e}=\cosh ^{-1} \frac{\gamma\left(1-\phi^{2}\right)+\left(1+\phi^{2}\right)}{2 \phi}
\end{aligned}
$$

with

$$
\gamma=\frac{r_{i}}{r_{e}}, \quad \phi=\frac{e}{r_{e}-r_{i}}=\frac{\bar{e} \gamma}{1-\gamma}
$$

where $\bar{e}=e / r_{i}$.

It was shown by Cairns et al. [1] that for this problem the velocity components $v_{1}$ and $v_{2}$ can be written as:

$$
v_{1}=0
$$

and

$$
v_{2}=\left(\cosh x_{2}-\cos x_{1}\right)^{2} \frac{1}{2 c \mu} \frac{p_{i}-p_{e}}{k_{e}-k_{i}}\left\{\left(\frac{b}{2}\right)^{2}-x_{\frac{2}{3}}\right\} .
$$

By substituting the velocities from equations (7) and (8) into the energy equation, equation (1) we get :*

$$
\begin{aligned}
\rho C_{p}\left(\cosh x_{2}-\cos x_{1}\right)^{2} \frac{1}{2 c \mu} \frac{p_{i}-p_{c}}{k_{e}-k_{i}}\left\{\left(\frac{b}{2}\right)^{2}-\right. & \left.x_{3}^{2}\right\} \frac{\partial T}{\partial x_{2}} \\
& =k \frac{\partial^{2} T}{\partial x_{3}^{2}} .
\end{aligned}
$$

Introducing the transformation

$$
\begin{gathered}
x_{2}^{\prime}=x_{2}-k_{1}, \quad \bar{x}_{3}=\frac{x_{3}}{(b / 2)}, \quad 0=\frac{T-T_{w}}{T_{0}-T_{w}}, \\
\bar{c}=\frac{c}{(b / 2)}, \quad \bar{P}_{e}=P e \frac{\ln (1 / \gamma)}{k_{i}-k_{e}} . \\
P_{e}=\frac{\rho C_{p}}{k \mu} \frac{1}{3} \frac{p_{i}-p_{e}}{\ln (1 / \gamma)}\left(\frac{b}{2}\right)^{2}
\end{gathered}
$$

and

$$
\bar{x}_{2}=-\int_{0}^{\bar{x}_{2}^{\prime}} \frac{\bar{c}^{2} \mathrm{~d} y_{2}}{\left[\cosh \left(y_{2}+k_{i}\right)-\cos x_{1}\right]^{2}} .
$$

Equation (9) becomes

$\frac{\partial^{2} \theta}{\partial \bar{x}_{3}^{2}}=\overline{P_{e}}\left(1-\bar{x}_{3}^{2}\right) \frac{\partial \theta}{\partial x_{2}}, \quad-1<\bar{x}_{3}<1, \quad \bar{x}_{2}>0$

subject to the boundary conditions

$$
x_{2}=0: \quad \theta=1 ; \quad x_{3}= \pm 1: \quad \theta=0 .
$$

Since equation (12) is linear, we assume a solution as :

$$
\theta\left(\bar{x}_{2}, x_{3}\right)=R\left(\bar{x}_{2}\right) Z\left(\bar{x}_{3}\right) \text {. }
$$

By using the technique of separation of variables, equation (1.3) is substituted into equation (12) and we get:

$$
\frac{3 \overline{P e}}{2} \frac{R^{\prime}}{R}=\frac{Z^{\prime \prime}}{\left(1-\bar{x}_{3}^{2}\right) Z}=-\lambda^{2}
$$

where $\lambda^{2}$ is an arbitrary constant. The first equation in equation (14) gives the general solution:

$$
R\left(\bar{x}_{2}\right)=C_{1} \exp \left(-\frac{2 \hat{\lambda}^{2}}{3 \bar{P} \bar{e}} \bar{x}_{2}\right)
$$

where $C_{1}$ is a constant to be determined by the boundary condition $\theta\left(0, \bar{x}_{3}\right)=1$ and the eigenvalue $\lambda^{2}$. The second equation from equation (14) is :

$$
Z^{\prime \prime}+\lambda^{2}\left(1-\bar{x}_{3}^{2}\right) Z=0
$$

subject to the boundary conditions $: Z( \pm 1)=0$.

* $\cosh x_{2}-\cos x_{1} \neq 0$ since $\cosh x_{2}>\cos x_{1}$ for the region $0 \leqslant x_{1} \leqslant 2 \pi$ and $0<k_{e} \leqslant x_{2} \leqslant k_{i}$, in this problem. 
Equation (16), which constitutes a Sturm-Lieuville eigenvalue problem, is solved approximately by the well-known Galerkin's method [2]. It is then combined with equation (15) to give the solution of equation (12) as

$$
\theta\left(\bar{x}_{2}, \bar{x}_{3}\right)=\sum_{i=1}^{N} C_{i}\left[\exp \left(-\frac{2 \lambda^{2} i}{3 \bar{P}_{e}} \bar{x}_{2}\right)\right] \bar{Y}_{i}
$$

where $C_{i}$ and $\bar{Y}_{i}$ are given by:

$$
C_{i}=\frac{8 \lambda_{i}}{\pi} \frac{\sum_{n=1}^{N}(-1)^{n+1} \frac{b_{n i}}{[(2 n-1 / 2) \pi]^{4}}}{\sum_{n=1}^{N} b_{n i}^{2}} \frac{b_{n i}}{2 n-1}
$$

and

$$
\bar{Y}_{i}=\frac{\sum_{n=1}^{N} \frac{b_{n i}}{(2 n-1)} \cos \frac{2 n-1}{2} \pi \bar{x}_{3}}{\sum_{n=1}^{N} \frac{b_{n i}}{(2 n-1)}}
$$

respectively. Also, $\lambda_{i}$ and $b_{n i}$ are the eigenvalue and the coefficients in the expansion of the eigenfunction respectively. Fifteen sets of values are computed in the numerical solution given below.

NUMERICAL SOLUTIONS AND DISCUSSIONS

The heat transfer is defined as

$$
\begin{aligned}
q_{w \bar{x}_{2}}= & -k\left(\frac{\partial T}{\partial x_{3}}\right)_{x_{3}=b / 2} \\
& =-\frac{k\left(T_{0}-T_{w}\right)}{b / 2}\left(\frac{\partial \theta}{\partial \bar{x}_{3}}\right)_{\bar{x}_{3}=1}=h_{\bar{x}_{2}}\left(T_{0}-T_{w}\right) .
\end{aligned}
$$

Thus, the local Nusselt number is given by:

$$
\begin{aligned}
& N u_{\bar{x}_{2}}=\frac{h_{\bar{x}_{2}}(b / 2)}{k}=-\left(\frac{\partial \theta}{\partial \bar{x}_{3}}\right)_{\bar{x}_{3}=1} \\
& =\sum_{i=1}^{N}\left\{C_{i}\left[\exp \left(-\frac{2 \lambda_{l}^{2}}{3 \bar{P} e} \bar{x}_{2}\right)\right] \sum_{m=1}^{N} b_{m i} \frac{2 m-1}{2} \pi(-1)^{m+1}\right\} .
\end{aligned}
$$

The average Nusselt number can be obtained by integrating over the disk surface, i.e.

$$
\begin{aligned}
\overrightarrow{N u}=-\frac{1}{\pi\left(\bar{r}_{e}^{2}-\bar{r}_{i}^{2}\right)} \int_{0}^{2 \pi k_{k_{t}}} N u_{\bar{x}_{2}} \bar{h}_{1} \bar{h}_{2} \mathrm{~d} x_{2} \mathrm{~d} x_{1} \\
=\frac{1}{\pi\left(\bar{r}_{e}^{2}-\bar{r}_{i}^{2}\right)} \int_{0}^{2 \pi} \int_{0}^{\pi} N \bar{x}_{z_{e}} \mathrm{~d} \bar{x}_{2} \mathrm{~d} x_{1} .
\end{aligned}
$$

Substituting $N u \bar{x}_{2}$ from equation (21) into equation (22), we get :

$$
\begin{aligned}
& \overline{N u}=\frac{1}{\pi\left(\bar{r}_{e}^{2}-\bar{r}_{i}^{2}\right)} \int_{0}^{2 \pi} \sum_{i=1}^{N}\left\{C_{i} \frac{3 \overline{P e}}{2 \lambda_{i}^{2}}\right. \\
& \left.\times\left[\exp \left(-\frac{2 \lambda_{i}^{2}}{3 \bar{P}_{e}} \bar{x}_{2 e}\right)-1\right] \sum_{m=1}^{N} b_{m i} \frac{2 m-1}{2} \pi(-1)^{m}\right\} \mathrm{d} x_{1} .
\end{aligned}
$$

The evaluation of the upper limit in the integration of $\mathrm{d} \bar{x}_{2}$ in equation (22), $\bar{x}_{2 e}$, is given in detail in Appendix $\mathrm{I}$.

Figures 2 and 3 are plots of the average Nusselt number as a function of $\bar{e}$ and $\gamma$ fo $\bar{r}_{i}=50$ and $P e=5000$ and 20000 , respectively. It is seen that in general the heat transfer is decreased if the inlet is eccentric, as compared with the heat transfer with concentric inlet under the same conditions.

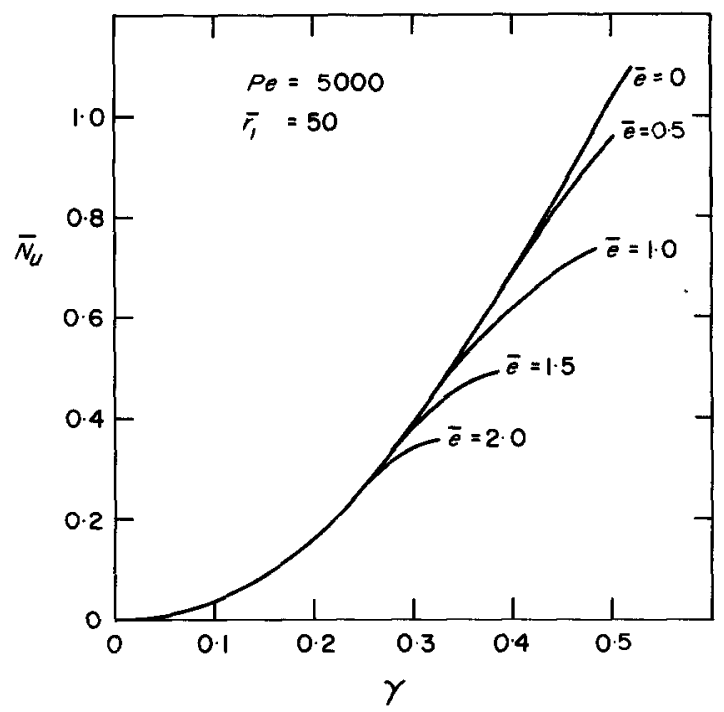

FIG. 2. Average Nusselt number vs. $\gamma(P e=5000)$.

The curves in Figs. 2 and 3 labeled $\bar{e}=0$, are for concentric inlet. Based on these figures, the following becomes apparent:

1. For a fixed eccentricity, $e$, and exit radius, $r_{e}$, increasing the inlet radius, $r_{1}$, will decrease the parameter $\bar{e}$ and increase the parameter $\gamma$. The average Nusselt number is thus increased.

2. For a pair of disks with fixed inlet and outlet radii, $r_{i}$ and $r_{e}$, the heat transfer is decreased if the eccentricity is increased.

3. For a pair of disks with fixed inlet radius, $r_{i}$, and eccentricity, $e$, the averaged Nusselt number is increased if the outlet radius $r_{e}$ is decreased.

4. As $\gamma$ is decreased, the curves approach to the concentric inlet curve. Since $\gamma$ is the ratio of $r_{i}$ to $r_{e}$ this means that 
the effect of eccentricity is reduced by either drilling a smaller hole or increasing the outlet radius.

5. The average Nusselt number is decreased for smaller Péclét numbers, which is physically reasonable since a smaller Péclét number means a larger thermal conductivity. For the same temperature difference, a decrease in heat transfer is expected.

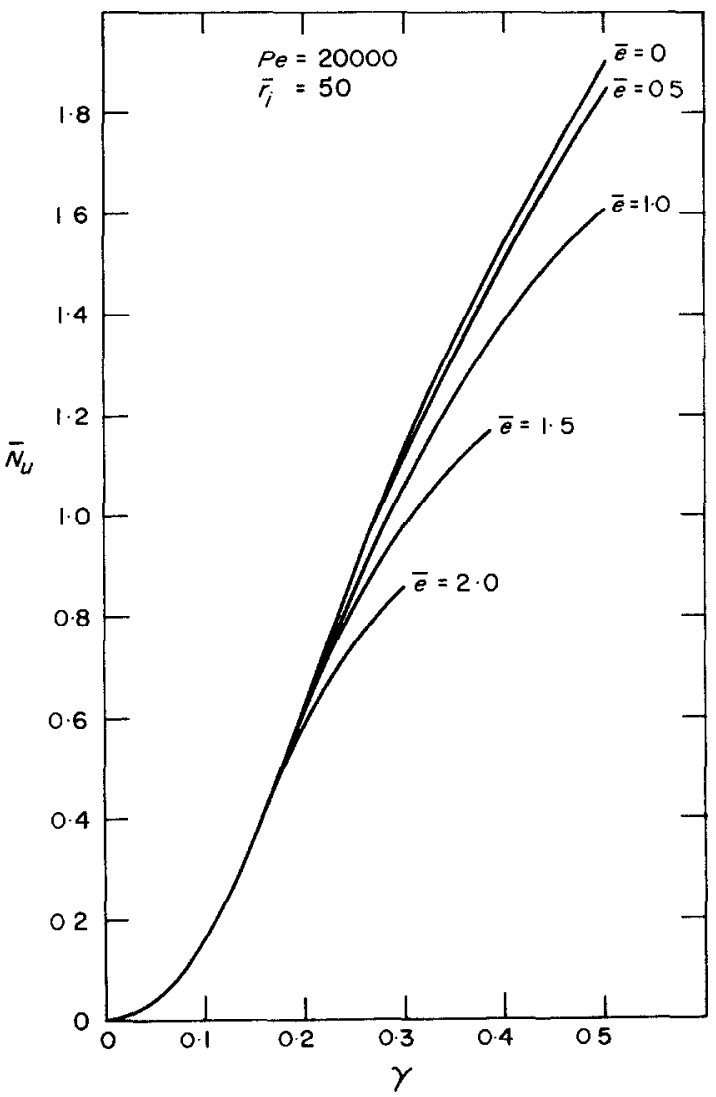

FIG. 3. Average Nusselt number vs. $\gamma\left(P e=2 \times 10^{5}\right)$.

\section{REFERENCES}

1. J. R. Cairns, M. M. Sibhom and T. Y. Na, Laminar creeping flow of incompressible fluids between parallel circular disks, ASME paper 68-FE-53, presented at the 1968 Fluid Engineering meeting, ASME, Philadelphia, Pa. (May 6-9 1968).
2. S. H. CRADAll, Engineering Analysis. McGraw-Hill, New York (1956).

\section{APPENDIX 1}

Evaluation of $x_{2}$.

The upper limit in the integration of $\mathrm{d} \bar{x}_{2}$ in equation (22), $\bar{x}_{2 e}$, can be obtained as follows: Integrating equation (11), wc get :

$$
\begin{aligned}
& \bar{x}_{2}=\frac{2 \bar{c}^{2}}{\sin ^{2} x_{1}}\left\{\frac{1-\mathrm{e}^{x_{2}} \cos x_{1}}{X\left(x_{2}\right)}-\frac{1-\mathrm{e}^{k_{i}} \cos x_{1}}{X\left(k_{i}\right)}\right. \\
& =\frac{\cos x_{1}}{\left|\sin x_{1}\right|}\left[\tan ^{-1}\left(\frac{\mathrm{e}^{x_{2}}-\cos x_{1}}{\left|\sin x_{1}\right|}\right)\right. \\
& \left.\left.-\tan ^{-1}\left(\frac{\mathrm{e}^{k_{1}}-\cos x_{1}}{\left|\sin x_{1}\right|}\right)\right]\right\}
\end{aligned}
$$

if $\sin x_{1} \neq 0$, and

$$
\begin{aligned}
\bar{x}_{2}=4 \bar{c}^{2}\left\{\left[\left(\mathrm{e}^{x_{2}}-1\right)^{-2}-\left(\mathrm{e}^{k_{i}}-1\right)^{-2}\right] / 2\right. \\
\left.+\left[\left(\mathrm{e}^{x_{2}}-1\right)^{-3}-\left(\mathrm{e}^{k_{1}}-1\right)^{-3}\right] / 3\right\}
\end{aligned}
$$

if $\sin x_{1}=0$, where

$$
X(x)=\mathrm{e}^{2 x}-2 \mathrm{e}^{x} \cos x_{1}+1 .
$$

At the inner radius, both equations give:

$$
\bar{x}_{2 i}=0 \text {. }
$$

At the outer radius, cquations (A.1) and (A.2) give:

$$
\begin{aligned}
& \bar{x}_{2 e}=\frac{2 \bar{c}^{2}}{\sin ^{2} x_{1}}\left\{\frac{1-\mathrm{e}^{k_{a}} \cos x_{1}}{X\left(k_{e}\right)}-\frac{1-\mathrm{e}^{k_{2}} \cos x_{1}}{X\left(k_{i}\right)}\right. \\
& -\frac{\cos x_{1}}{\left|\sin x_{1}\right|}\left[\tan ^{-1}\left(\frac{\mathrm{e}^{k_{0}}-\cos x_{1}}{\mid \sin x_{1}}\right)\right. \\
& \left.\left.-\tan ^{-1}\left(\frac{\mathrm{e}^{k_{i}}-\cos x_{1}}{\left|\sin x_{1}\right|}\right)\right]\right\}
\end{aligned}
$$

if $\sin x_{1} \neq 0$, and

$$
\begin{aligned}
\bar{x}_{2 e}=4 \bar{c}^{2}\left\{\left[\left(\mathrm{e}^{k_{e}}-1\right)^{-2}-\right.\right. & \left.\left(\mathrm{e}^{k_{1}}-1\right)^{-2}\right] / 2 \\
& \left.+\left[\left(\mathrm{e}^{k_{e}}-1\right)^{-3}-\left(\mathrm{e}^{k_{1}}-1\right)^{-3}\right] / 3\right\}
\end{aligned}
$$

if $\sin x_{1}=0$. 